\title{
Los proyectos de aula y el aprendizaje por investigación
}

\section{Liliana Hernández Romero 3}

lilihana@gmail.com

\section{Resumen}

El presente trabajo busca mostrar la importancia de los proyectos de aula para promover la investigación en la escuela; utiliza el aprendizaje por investigación como herramienta metodológica que permite a los estudiantes adquirir, comprender, aclarar y aplicar los conceptos alrededor del área de biotecnología, gracias a que ellos mismos desarrollan los proyectos.

Este tipo de actividad genera una visión diferente hacia las temáticas tratadas en el aula, desarrollando y fortaleciendo sus competencias interpretativas, propositivas y argumentativas.

\section{Palabras clave}

Proyectos de aula, Aprendizaje por investigación, Aprendizaje significativo, Enseñanza-Aprendizaje, Competencias.

3. Estudiante del Departamento de Química - UPN 


\section{Introducción}

Se ha demostrado que la educación que se implementa en muchas instituciones conlleva al estudiante a seguir esquemas repetitivos de contenidos, desconoce su realidad y sus intereses, sin lograr generar realmente un aprendizaje significativo, lo que limita de forma determinante la capacidad productiva, analítica, propositiva y argumentativa de los estudiantes. (Universidad Pedagógica Nacional, 2005).

Por tal motivo, es importante y necesario promover espacios en el proceso de enseñanzaaprendizaje, que involucren diferentes metodologías y que, sobre todo, le permita a los estudiantes desarrollar habilidades en las competencias propositivas, interpretativas $y$ argumentativas.

La calidad en la educación representa todo un reto para los profesores; por lo tanto, para su mejoramiento es necesario que el proceso de enseñanza-aprendizaje esté acompañado de procesos de investigación, generando así alternativas para este. Así, la enseñanza de las ciencias debe enfocarse en promover la formación investigativa, pues día a día se generan avances científicos y tecnológicos, lo que implica el mejoramiento en las herramientas pedagógicas y didácticas a utilizar en los procesos de enseñanza. En consecuencia, la investigación es una herramienta útil para mejorar dichos procesos. En este artículo se quiere mostrar la importancia de los proyectos de aula utilizados como herramientas para promover la investigación pedagógica a partir de problemáticas cotidianas.

\section{Marco teórico}

Para el desarrollo de esta propuesta, los proyectos de aula fueron entendidos como actividades encaminadas a la resolución de problemas que despiertan interés y surgen por común acuerdo; durante este proceso los estudiantes junto con sus profesores buscan darle significado a problemas específicos.

Por otra parte, es importante mencionar brevemente dos modelos pedagógicos de aprendizaje:

\section{Aprendizaje por investigación}

Este plantea cómo la investigación es un proceso de construcción de conocimientos y actitudes (Cañal y Porlan), que posibilita el cuestionamiento y la búsqueda de explicaciones por parte del individuo. La construcción de conocimiento está basada en la construcción interior de representaciones e interpretaciones por parte de los sujetos, existen ideas previas en los estudiantes y estas son de carácter modificable, el aprendizaje de conocimientos debe ser concebido como la construcción de relaciones y significados (Salcedo y García, 1995).

\section{Teoría de la asimilación de Ausubel}

Esta teoría hace énfasis en los organizadores previos y otras condiciones para un aprendizaje significativo. El alumno aprende cuando es capaz de atribuir significado al contenido de lo que está estudiando; es decir, cuando es capaz de construir un esquema de conocimiento relativo a este contenido. Esto se hace posible a partir de la interacción del alumno, los conceptos y el profesor.

Además, se debe tener en cuenta que las competencias se integran como un conjunto de elementos cognitivos, técnicos, afectivos y sociales que nos acercan a una forma educativa más íntegra. 
La competencia propositiva implica

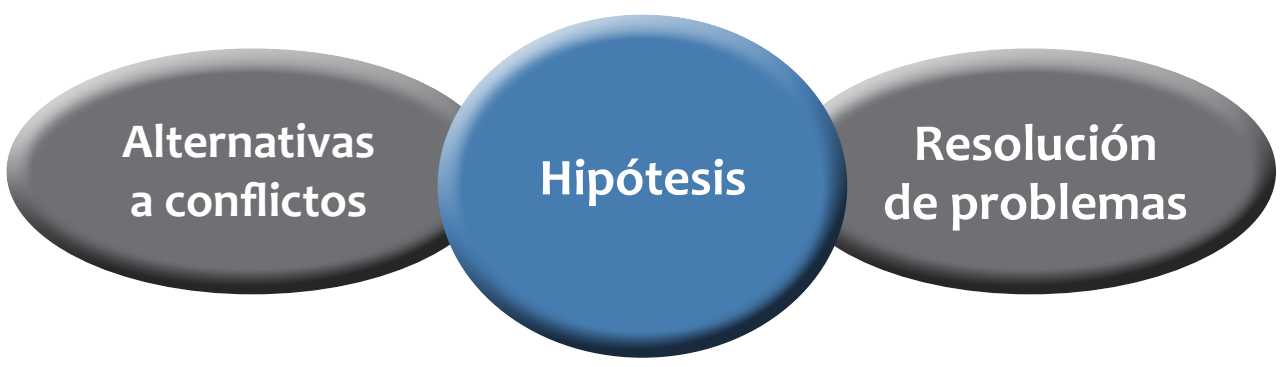

La competencia argumentativa lleva a una articulación entre

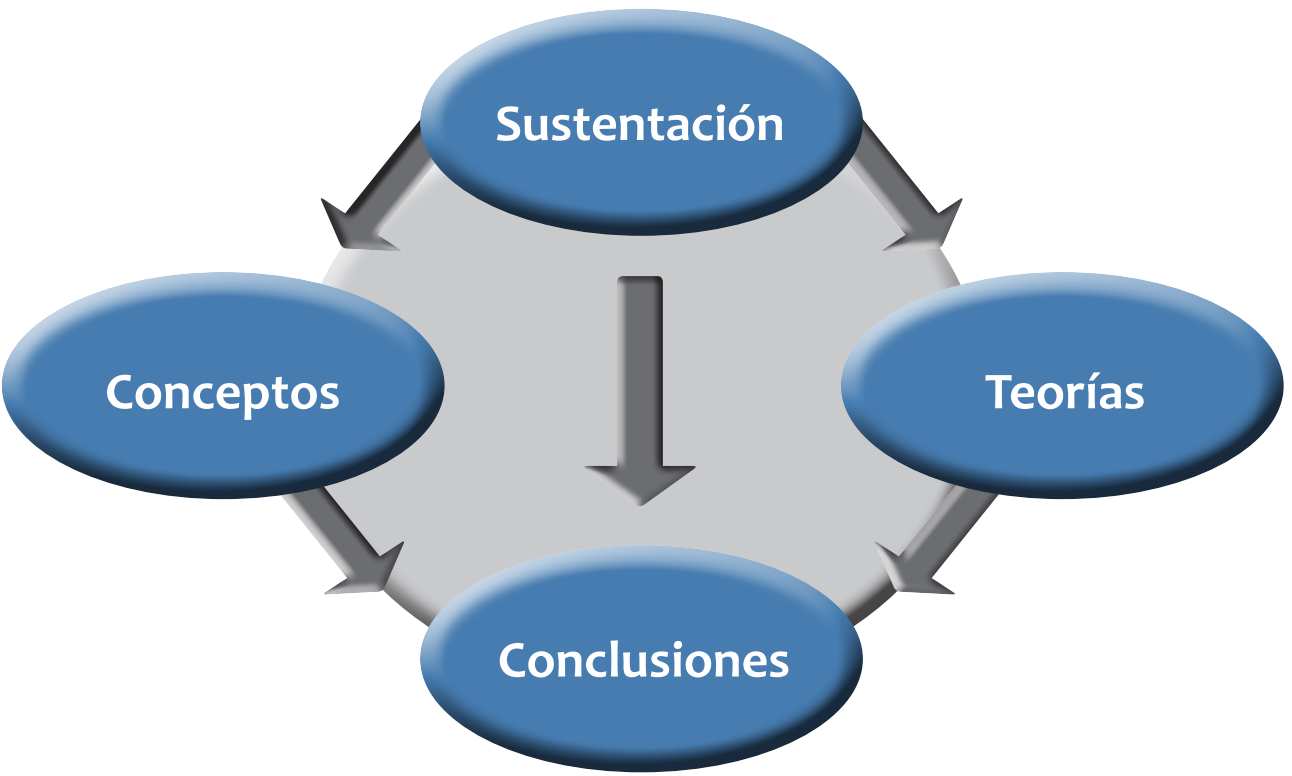

La competencia interpretativa es la capacidad de

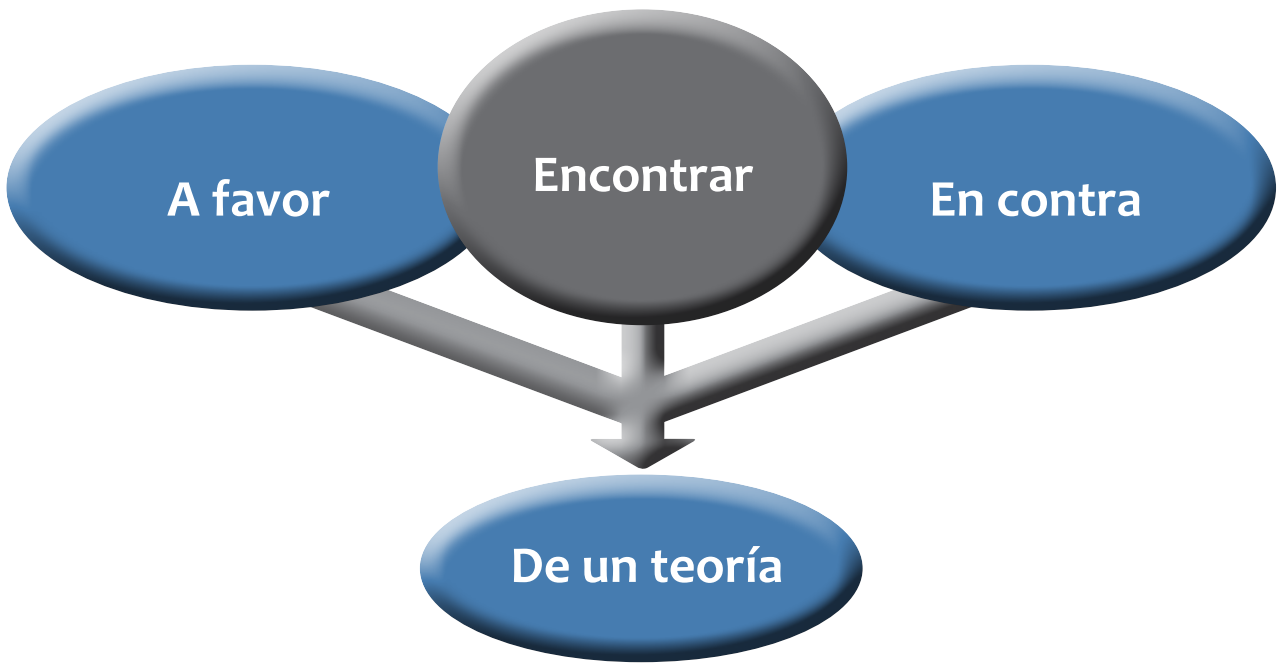




\section{Metodología}

Datos de

identificación:
Colegio: Instituto Pedagógico Nacional (IPN)

Grado 110: 11-04

Número de estudiantes: 33

Grupo piloto: 4 estudiantes
Técnicas e

instrumento de

recolección de

información:

\section{Técnicas e instrumento de recolección de información:}

Guías de laboratorio

Actividades en clase

Consultas extra-clase

Prácticas de laboratorio

Exposición de los avances y del proyecto final

Prueba final escrita por grupos de trabajo
Estrategia metodológica:
1. Se seleccionó un grupo piloto conformado por 4 estudiantes. Su proyecto llevaba por título Extracción de los 6-carotenos del achiote (bixa orellana) con fines terapéuticos.

2. Se facilitó información y se explicaron algunos conceptos teóricos necesarios para lograr un buen desempeño en el desarrollo del trabajo, principalmente en las prácticas de laboratorio.

3. Se realizaron 2 talleres relacionados con la parte teórico-experimental.

De manera general, las pruebas constaban de cuatro tipos de pregunta: de selección múltiple, relación de términos, verdadero o falso y de respuesta abierta. La intención de estas preguntas era evaluar qué conceptos tenían claros después de haber realizado las prácticas de laboratorio y, además, conocer si habían realizado consultas con respecto a la temática tratada, pues algunas de las preguntas estaban relacionadas con conceptos que no habían sido mencionados en la teoría, ni durante la práctica. Por último, se realizaron preguntas de tipo abierto, cuyo propósito era que relacionaran conceptos teóricos y prácticos con la vida cotidiana.

4. Finalmente, se realizó una prueba final escrita, tanto para el grupo piloto, como para los demás grupos del salón; todas las pruebas presentaban los mismos tipos de pregunta descritos anteriormente. 


\section{Resultados}

A partir de los resultados grupales se puede decir que, en general, todos los grupos presentan un aprendizaje de conceptos relacionados con sus proyectos; sin embargo, se nota que para un buen desarrollo de estos es necesario realizar consultas extra-clase, ya que esto permite a los estudiantes mejorar su conocimiento y, así mismo, vincular los conocimientos con la vida cotidiana; es decir, el aprendizaje por investigación les permite a los estudiantes construir y relacionar los conceptos tratados en la teoría con la práctica.

Adicionalmente, se identificó que el desarrollo de proyectos les permite fortalecer las competencias propositivas, interpretativas y argumentativas gracias específicamente a las prácticas de laboratorio y exposiciones.

Así mismo, se puede observar que es necesario que los profesores sean muy dedicados a los grupos, ya que esto estimula y genera mayor interés en el momento de realizar consultas, prácticas de laboratorio, exposiciones, etc.

\section{Conclusiones}

* El desarrollo de proyectos les permite a los estudiantes aclarar, comprender y construir conceptos de forma adecuada.

* El proceso de enseñanza-aprendizaje es más enriquecedor si los estudiantes logran relacionar las temáticas de la clase con problemas de la vida cotidiana.

* El aprendizaje por investigación permite cambiar las metodologías aplicadas en el aula cotidianamente.

* Las prácticas de laboratorio permiten que los estudiantes comprendan fenómenos, lo cual no se logra sólo desde la teoría.

* El desarrollo de proyectos de aula posibilita a los estudiantes mejorar sus competencias argumentativas, interpretativas y propositivas.

* El aprendizaje por investigación y los proyectos de aula son herramientas pedagógicas alternativas útiles en la labor docente.

\section{Bibliografía}

Arteta, J. Ibáñez, X. Martínez, S. y Pedraza, M. (2004). La investigación como espacio de calificación docente y de aprendizaje de las ciencias en educación básica. Revista de la Asociación Colombiana de Ciencias Biológicas. 16(1), enero-junio.

Ausubel, D. (1981). Psicología educativa: un punto de vista cognoscitivo. México: Editorial Trillas.

Bogoya, D. Restrepo, G. y Vinnet, M. (2000). Competencias y proyecto pedagógico. Bogotá: Editorial UNILIBROS.

Cerda, H. (2000). La evaluación como experiencia total. Bogotá: Editorial Magisterio.

Cerda, H. (2003a). La nueva evaluación educativa: desempeños, logros competencias y estándares (actualización pedagógica). Bogotá: Editorial Magisterio. Primera edición.

Cerda, H. (2003b). La nueva evaluación educativa. Bogotá: Editorial Magisterio.

Novac, J. y Gowin, D. (1988). Aprendiendo a aprender. Barcelona, España: Martínez Roca Editores.

Segura, D. (1999). La construcción de la confianza una experiencia en proyectos de aula. Bogotá: Escuela Pedagógica Experimental - IDEP. de desarrollo Institucional. Bogotá. 